\title{
Goldmann tonometry tear film error and partial correction with a shaped applanation surface
}

This article was published in the following Dove Press journal:

Clinical Ophthalmology

\author{
Sean J McCafferty ${ }^{1-4}$ \\ Eniko T Enikov ${ }^{5}$ \\ Jim Schwiegerling ${ }^{2,3}$ \\ Sean M Ashley ${ }^{1,3}$ \\ 'Intuor Technologies, ${ }^{2}$ Department \\ of Ophthalmology, University of \\ Arizona College of Medicine, \\ ${ }^{3}$ University of Arizona College \\ of Optical Science, ${ }^{4}$ Arizona Eye \\ Consultants, ${ }^{5}$ Department of \\ Mechanical and Aerospace, University \\ of Arizona College of Engineering, \\ Tucson, AZ, USA
}

Purpose: The aim of the study was to quantify the isolated tear film adhesion error in a Goldmann applanation tonometer (GAT) prism and in a correcting applanation tonometry surface (CATS) prism.

Methods: The separation force of a tonometer prism adhered by a tear film to a simulated cornea was measured to quantify an isolated tear film adhesion force. Acrylic hemispheres (7.8 mm radius) used as corneas were lathed over the apical $3.06 \mathrm{~mm}$ diameter to simulate full applanation contact with the prism surface for both GAT and CATS prisms. Tear film separation measurements were completed with both an artificial tear and fluorescein solutions as a fluid bridge. The applanation mire thicknesses were measured and correlated with the tear film separation measurements. Human cadaver eyes were used to validate simulated cornea tear film separation measurement differences between the GAT and CATS prisms.

Results: The CATS prism tear film adhesion error $(2.74 \pm 0.21 \mathrm{mmHg})$ was significantly less than the GAT prism $(4.57 \pm 0.18 \mathrm{mmHg}, p<0.001)$. Tear film adhesion error was independent of applanation mire thickness $\left(R^{2}=0.09, p=0.04\right)$. Fluorescein produces more tear film error than artificial tears $(+0.51 \pm 0.04 \mathrm{mmHg} ; p<0.001)$. Cadaver eye validation indicated the CATS prism's tear film adhesion error $(1.40 \pm 0.51 \mathrm{mmHg})$ was significantly less than that of the GAT prism $(3.30 \pm 0.38 \mathrm{mmHg} ; p=0.002)$.

Conclusion: Measured GAT tear film adhesion error is more than previously predicted. A CATS prism significantly reduced tear film adhesion error by $\sim 41 \%$. Fluorescein solution increases the tear film adhesion compared to artificial tears, while mire thickness has a negligible effect.

Keywords: glaucoma, intraocular pressure, IOP, Goldmann, bias, error, tonometer, applanation, tear film

\section{Introduction}

Goldmann applanation tonometry remains the gold standard for intraocular pressure (IOP) measurement, although numerous errors have occurred due to variability in corneal geometry, biomechanics, and tear film. ${ }^{1-7}$ Most eye care professionals have only accepted corrections for central corneal thickness (CCT), which has been shown to be an incomplete correction for total Goldmann applanation tonometry error and of questionable utility without corrections for corneal curvature and corneal rigidity. ${ }^{8,9}$ Goldmann applanation tonometry significantly underestimates true intracameral IOP measured by a pressure transducer in both live human eyes and human cadaver eyes. $^{7,10-13}$ While the corneal biomechanical parameters increasing corneal rigidity tend toward an overestimation of applanation IOP, the tear film adhesion partly negates these errors by an effective reduction in applanation IOP measurement. The tear film error is estimated to be between 0.330 and $0.415 \mathrm{~g}$ of force in Goldmann applanation tonometry, which translates to a $3.30-4.15 \mathrm{mmHg}$ lower measured IOP. ${ }^{14,15}$
Correspondence: Sean J McCafferty Intuor Technologies, LLC 6422 E. Speedway Boulevard, Suite 100 , Tucson, AZ 857I0, USA

Tel +l 5203273487

Email sjmccaff@email.arizona.edu 
Mathematical modeling has demonstrated the parameters affecting tear film adhesion to include the average contact angle between the cornea and the applanation surface over the tear film meniscus (a more acute angle increases the capillary adhesion), the linear circumference of the tear film meniscus, and the surface tension of the fluid bridge between the two contacting solids. ${ }^{14,16-18}$ The following equation is the mathematical model of the tonometer prism surface with the tear film of the cornea during full applanation:

$$
F=\pi * \rho^{*} \sigma *(2 * \sin (\alpha+\theta)+\sin (\alpha) *(R / r-R / l))
$$

where $F$ denotes the tear film adhesion force $(\mathrm{N}) ; \rho$ denotes the cylindrical radius of contact ( $\mathrm{mm}$ ); $\sigma$ denotes the surface tension (N/mm); $\alpha$ denotes the average angle between the two solid bodies over the fluid bridge (rad.); $\theta$ denotes the angle of contact incidence (rad.); $R$ denotes the effective corneal curvature $(\mathrm{mm}) ; r$ denotes the radius of curvature of fluid bridge $(\mathrm{mm})$; and $l$ denotes the radius of fluid bridge $(\mathrm{mm})$.

The model parameters are depicted in Figure 1. The modeling predicted an $\sim 45 \%$ reduction in tear film adhesion error by increasing the contact angle $(\theta)$ between the cornea and the applanating surface from $5^{\circ}$ in the Goldmann applanation tonometer (GAT) prism to $25^{\circ}$ in the correcting applanation tonometry surface (CATS) prism. ${ }^{14}$ The present study was designed to quantify and compare the tear film adhesion error in the Goldmann (GAT) prism to a modified applanation surface (CATS) prism.

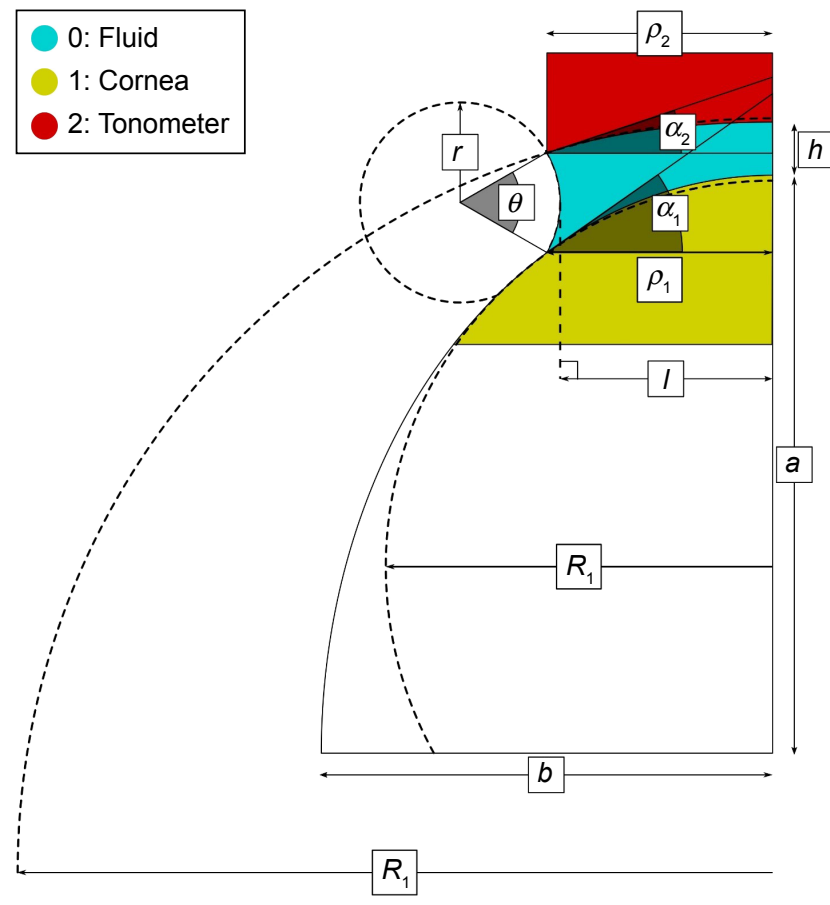

Figure I Schematic of tear film adhesion modeling.

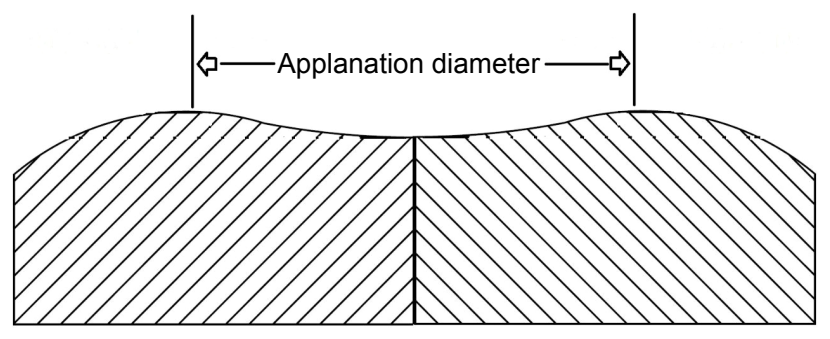

Figure 2 CATS tonometer prism cross section of the modified applanating surface. Abbreviation: CATS, correcting applanation tonometry surface.

\section{Methods}

The CATS tonometer prism is a replacement modified GAT prism which optimizes the corneal applanating surface from a flat surface to a central concave and a peripherally convex surface as illustrated in Figure 2 and photographed in Figure 3. The prism was designed to decrease the sensitivity of applanation tonometry to corneal biomechanical variability as well as tear film adhesion error. ${ }^{14}$

In mathematical modeling, the CATS tonometer prism reduces GAT tear film measurement error by $\sim 45 \% .{ }^{14}$ The two geometric factors, illustrated in Figure 4, which influence the tear film adhesion force and subsequent error are the angle $\theta$ between the cornea and prism applanation surface bridged by the tear film meniscus and the circumference of the applanation diameter (ie, $-\pi \times 3.06 \mathrm{~mm}=9.61 \mathrm{~mm}$ ). ${ }^{16,17}$

Tear film adhesion was measured by examining the force required to separate two bodies (prism and simulated cornea) adhered by an artificial tear film bridge as illustrated in

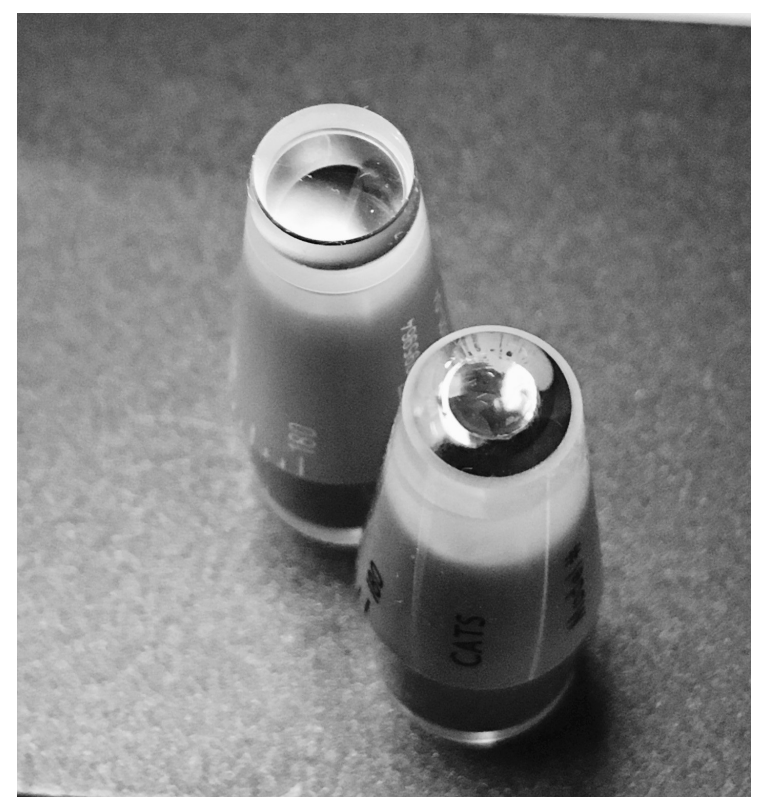

Figure 3 Photograph of the CATS tonometer prism and GAT prism applanating surfaces.

Abbreviations: CATS, correcting applanation tonometry surface; GAT, Goldmann applanation tonometer. 
Figure 4 (upper right). A bench top study was completed using $7.8 \mathrm{~mm}$ radius polymethyl methacrylate (PMMA) acrylic hemispheres as simulated corneas. The simulated acrylic corneas were lathed flat on their apical surface to a diameter of $3.06 \mathrm{~mm}$ for use with the GAT prism. Simulated corneas were also lathed to the inverse of the CATS prism surface over the applanation area for CATS tear film separation measurements. This was completed to simulate the isolated tear film adhesion force at full applanation for each prism. The separation force was recorded at the moment the simulated acrylic cornea and tonometer prism connected by a tear film fluid bridge were pulled apart.

Simulated acrylic corneas were used primarily to quantify the isolated tear film adhesion force. Under these testing conditions, the use of human cadaver eyes cannot isolate the tear film adhesion force. The separation of a prism from a manometrically fluid-filled cadaver eye simultaneously measures both the tear film adhesion and the IOP force on the prism face over a dynamically reducing applanation area as the prism separates. For this reason, as the IOP increases, the tear film adhesion separation force decreases because it is no longer measuring at full applanation but at a reducing area of applanation. If it were possible to measure the tear film separation force on a cadaver eye at an IOP of $0.0 \mathrm{mmHg}$ then this would negate the error, but the human globe will collapse and fail to applanate under these conditions. Therefore, this dynamic prism adhesion separation process using cadaver eyes is not suitable to measure the isolated tear film separation at full applanation and indicates a falsely low separation force. At low IOPs ( $5 \mathrm{mmHg}$ ), cadaver eyes begin to approximate the static conditions needed to measure the tear film separation force, but it remains a dynamic separation process. As a result, human cadaver eyes at 20,10, and $5 \mathrm{mmHg}$ IOP were used to extrapolate what the tear film adhesion force would be if the IOP was zero (0) $\mathrm{mmHg}$. These extrapolated values were used to validate the comparative difference in tear film separation force on human corneas between the CATS and GAT prisms.

Both simulated acrylic corneas and human cadaver globes were used to measure and validate the tear film separation force using a calibrated Perkins (Goldmann-type) tonometer (Perkins Tonometer MK2; Haag-Streit, Mason, OH, USA) by applying one of the two bridging fluids to the corneal surface prior to each measurement: 1) fluorescein (fluorescein sodium ophthalmic solution $0.25 \% / 0.4 \%$; Bausch \& Lomb Incorporated, Bridgewater, NJ, USA) or 2) artificial tears (Allergan, Inc., Irvine, CA, USA) with buffered sterile saline having an osmolality of $290 \mathrm{mOsm} / \mathrm{kg}$, with ingredients including sodium chloride, boric acid, calcium chloride, magnesium chloride, potassium chloride, purified water, sodium borate, and carboxymethyl cellulose sodium.

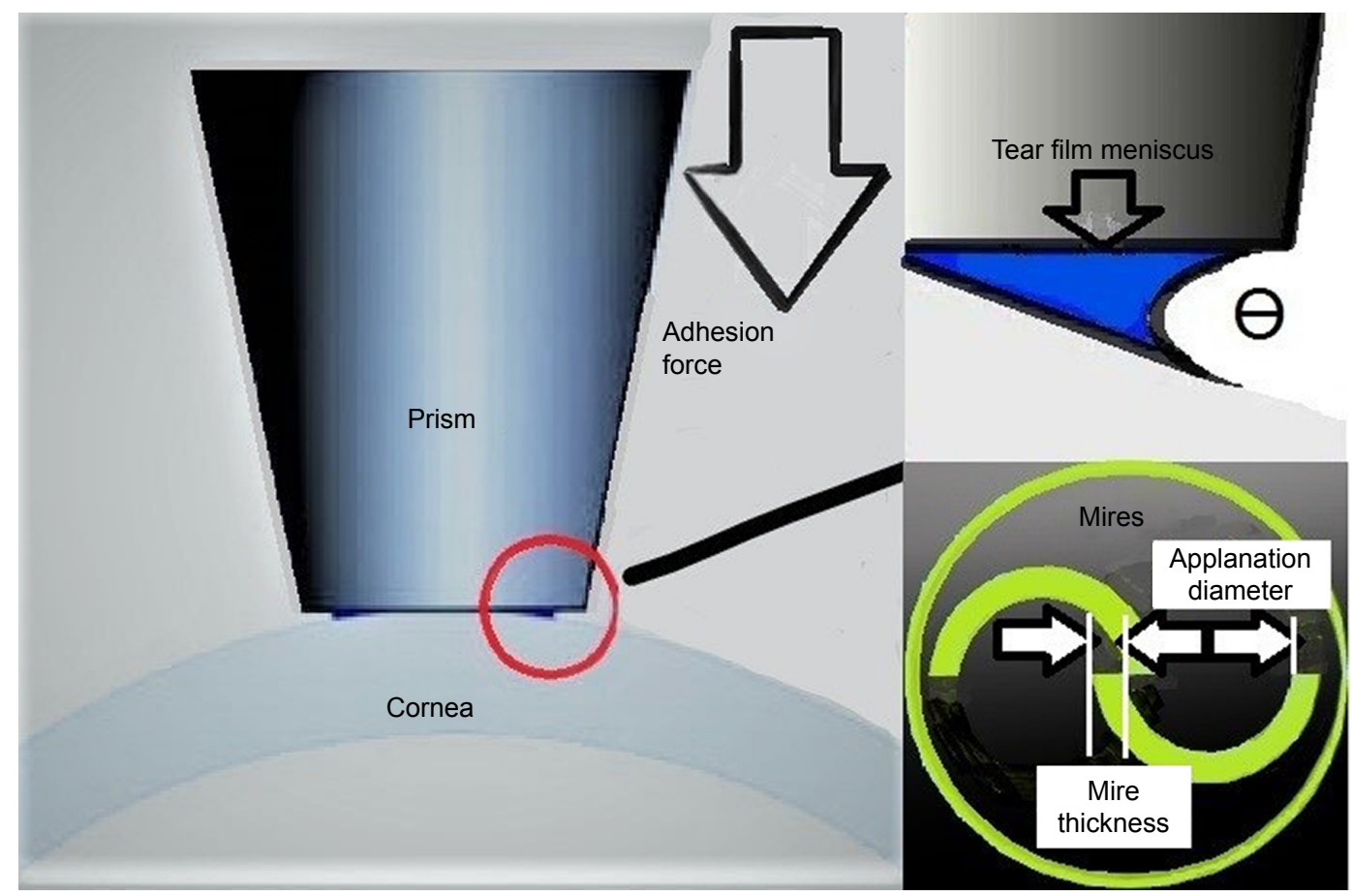

Figure 4 Illustration of Goldmann applanation tonometry tear film adhesion.

Notes: Upper right inset: illustration of tear film meniscus and angle theta between the cornea and the prism applanation surface. Lower right inset: illustration of applanation mires imaged through the tonometer prism demonstrating mire thickness and measurement applanation end point. 
The separation force was measured on a calibrated scale (WeighMax, model NJ-100; Beijing, China) tared between each of the 10 measurements. The tonometer applanation force was reduced at a rate of $0.5 \mathrm{~g} / \mathrm{min}$ until prism-corneal separation. The separation force measured on the scale was recorded at the time of prism contact separation from the corneal surface (simulated acrylic cornea or cadaver cornea). Figure 5 is a photograph of the PMMA acrylic hemisphere adhesion force test apparatus. The applanation mire thickness illustrated in Figure 4 (lower right) was measured by imaging mires through a microscope (Amscope, model 12-3; Irvine, CA, USA). The microscope image was also used to ensure complete and centered applanation between the cornea (both acrylic cornea and cadaver cornea) and the tonometer prism for accurate tear film separation force measurement.

A separate set of tests was completed on two fresh cadaver eyes (Georgia Eye Bank, Atlanta, GA, USA). Figure 6 is a photograph of the apparatus for use with pressurized cadaver eyes, which uses a conceptually identical measurement process as the acrylic corneas. The whole globes were shipped $<24$ hours postmortem and stored at $4^{\circ} \mathrm{C}$ in Optisol chambers until use. ${ }^{19}$ All corneas were of corneal transplant quality without prior surgery. The cadaver eyes are used on the day of arrival within 36 hours postmortem.

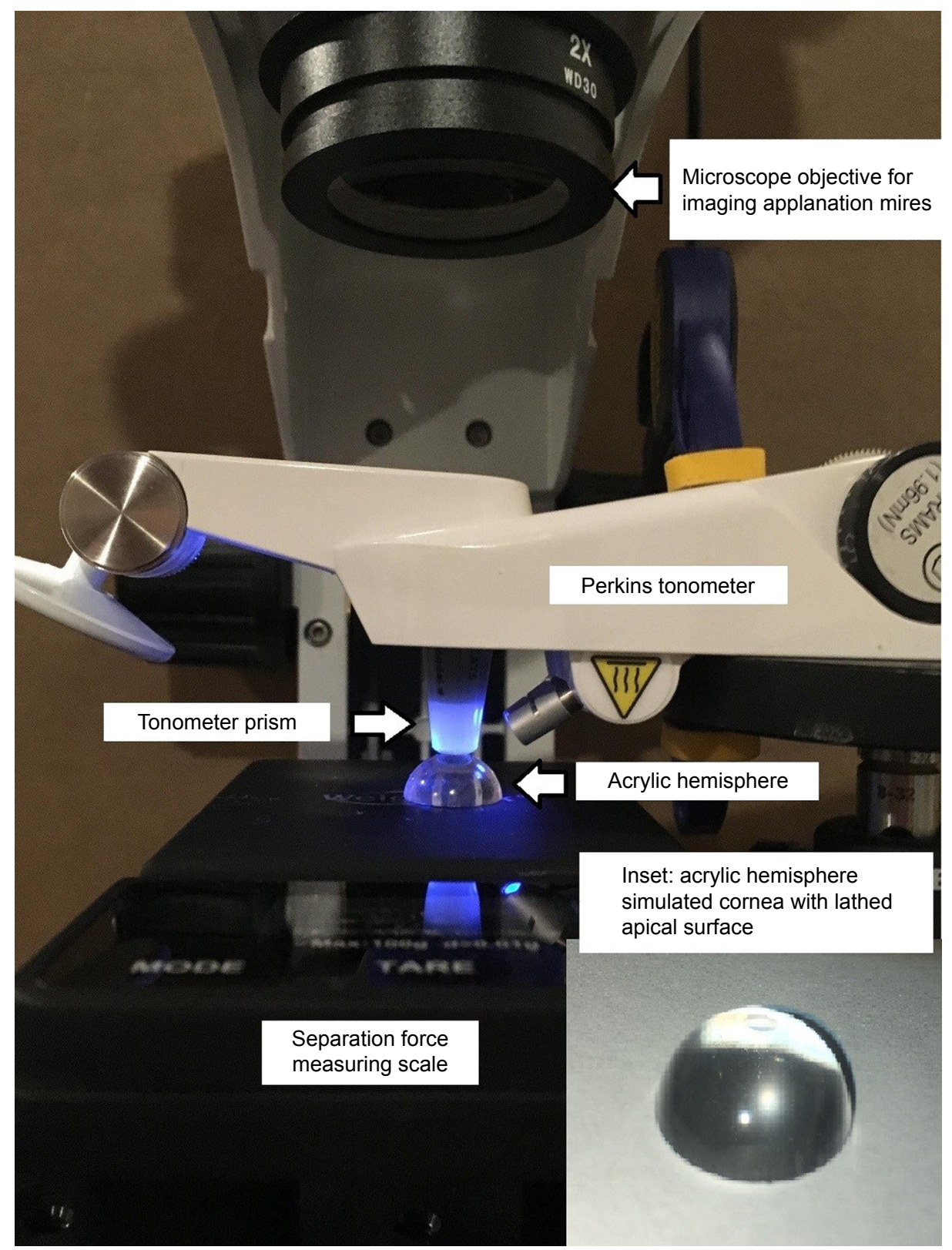

Figure 5 Photograph of applanation tonometry tear film adhesion measurement setup with Perkins tonometer, acrylic hemisphere, scale, and mire imaging microscope. 
Eyes with a history or evidence of previous anterior segment intraocular surgery (except cataract) or corneal abnormalities were excluded.

They were stabilized in a specially designed apparatus for manometrically pressurizing and measuring IOP on a whole globe (Figure 6) with the cornea exposed. A 22 G needle with Y-adaptor (Dickinson and Company, Franklin Lakes, NJ, USA) was inserted into the anterior chamber via a scleral approach. The needle intravenous tube was connected to a manometric transducer (Dwyer Instruments, Michigan, IN, USA), an isotonic sodium chloride solution infusion bottle, and an open-air reference tube. The transducer was zeroed with an open tube reference, and indicated pressures were verified by open manometry tube height. The IOP was set manometrically to 5,10 , and $20 \mathrm{mmHg}$ and confirmed via the pressure transducer. The globe elevation at the central cornea was maintained equal in all measurements to ensure a constant intracameral IOP.

Measurements were completed with the PMMA hemispheres using both the CATS and GAT prisms and using both artificial tear solution and fluorescein. Mire thickness measurements were completed on only the fluorescein tests. Ten separate measurements were made on each of the acrylic simulated corneas and at each manometrically set pressure on each of the cadaver eyes (140 measurements total). Each set of 10 measurements was averaged with a standard deviation. Statistical significance using the acrylic corneas was examined with general linear mixed-effects (GLME) modeling variables, CATS prism, GAT prism, mire thickness, artificial tears, fluorescein, and combined interactions. Statistical significance with cadaver eyes also included IOP (both first- and second-order IOP effects) and random effects. The difference

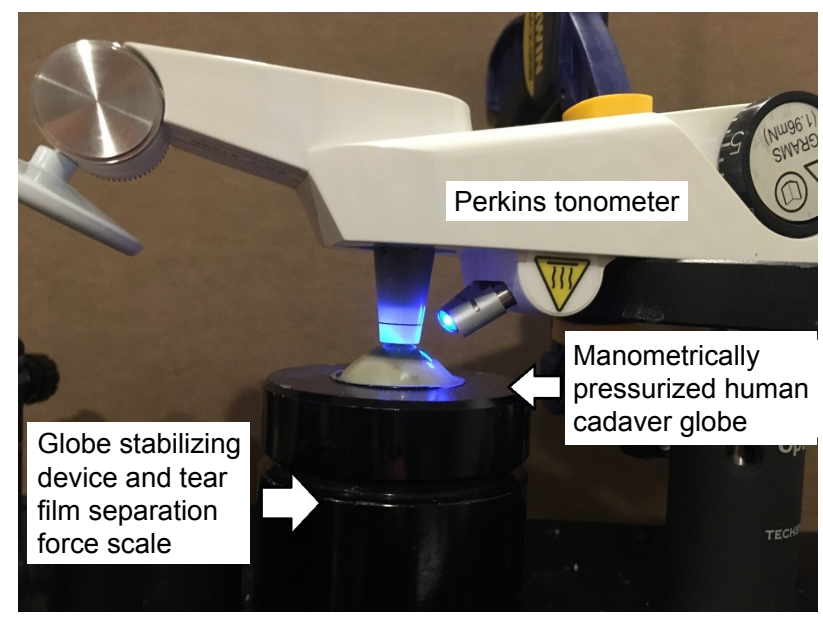

Figure 6 Photograph of applanation tonometry tear film adhesion measurement setup with Perkins tonometer and cadaver eye apparatus. in the mean values between CATS and GAT, as well as the $p$-value corresponding to a two-sample difference-of-mean $t$-test, was examined.

\section{Results}

The GAT prism has a significant tear film adhesion error of $4.57 \pm 0.18 \mathrm{mmHg}$ at full applanation when tested using the simulated PMMA hemisphere corneas. The CATS prism tear film adhesion error was significantly less than the GAT prism at $2.74 \pm 0.21 \mathrm{mmHg}, p<0.001$. The difference between the two prisms is illustrated in Figure 7.

The resultant fit from the GLME analysis on the cadaver eye data is as follows:

$$
\begin{aligned}
\hat{\mathrm{y}} \text { (tear film error) } & \\
= & 3.28[\mathrm{mmHg}]-1.915[\mathrm{mmHg}] \cdot \mathrm{CATS} \\
& -(0.26-0.229 \cdot \mathrm{CATS}) \mathrm{IOP}+0.0086 \cdot \mathrm{IOP}^{2}
\end{aligned}
$$

where CATS indicates the tonometer used $(\mathrm{GAT}=0$, CATS =1).

Validation with the human cadaver eyes extrapolating the measured tear film adhesion force at zero $(0 \mathrm{mmHg})$ from the 20,10, and $5 \mathrm{mmHg}$ intracameral IOP separation measurements (Figure 8) indicated that the CATS prism tear film adhesion error of $1.40 \pm 0.51 \mathrm{mmHg}$ was significantly less than that of the GAT prism of $3.30 \pm 0.58 \mathrm{mmHg}, p=0.002$.

The tear film adhesion error does not correlate significantly with applanation mire thickness measurements $\left(R^{2}=0.09\right.$,

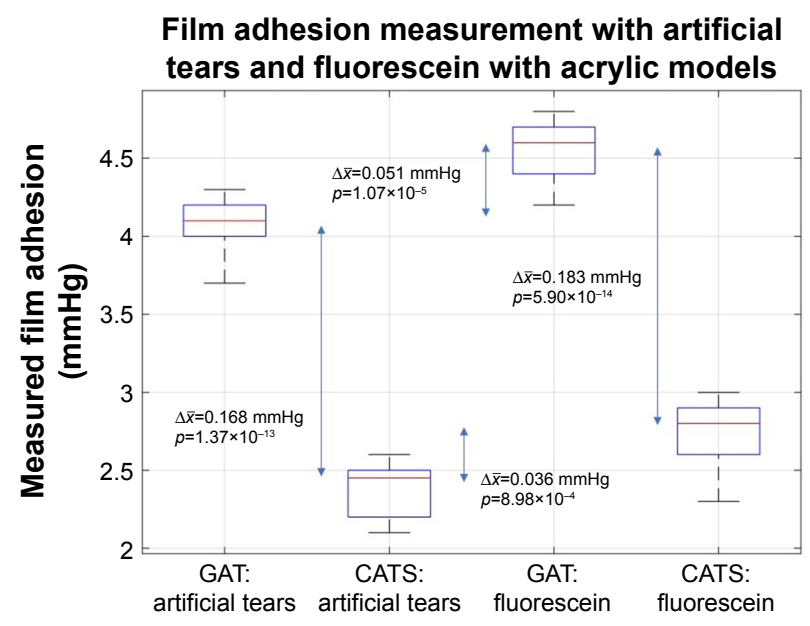

Figure 7 Tear film adhesion force error $(\mathrm{mmHg})$ comparing the CATS and GAT prisms on PMMA-simulated cornea.

Notes: Box-and-whisker plots of measured film adhesion for when using artificial tears and fluorescein as tear film stimulants and when using the GAT and CATS tonometers. The figure also includes the difference of the mean values between groups and the $p$-value corresponding to a two-sample difference of mean $t$-test.

Abbreviations: CATS, correcting applanation tonometry surface; GAT, Goldmann applanation tonometer; PMMA, polymethyl methacrylate. 


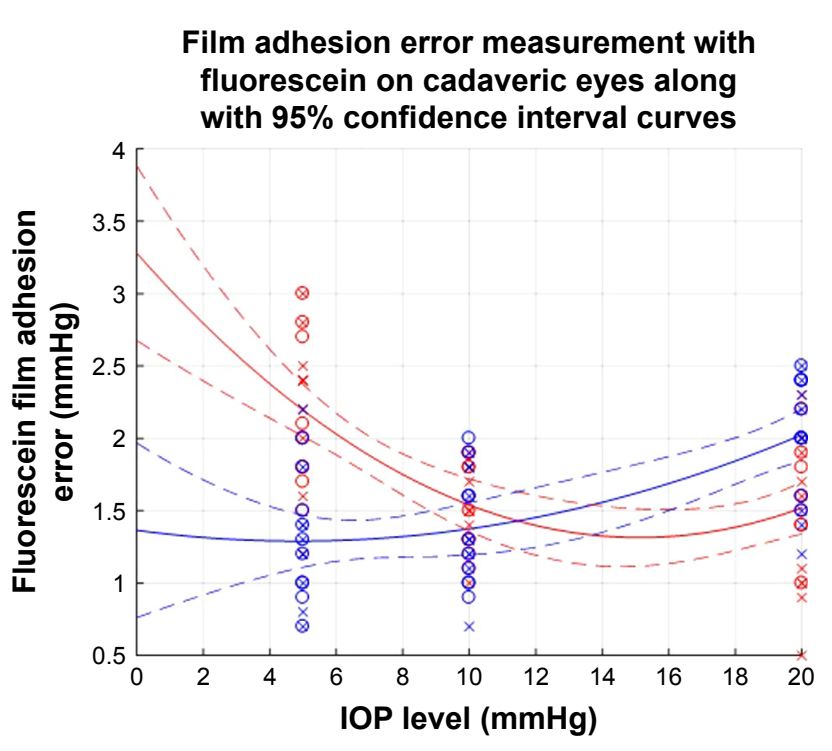

Figure 8 Cadaver globe tear film adhesion force error $(\mathrm{mmHg})$ comparing the CATS (blue) and GAT (red) prisms demonstrating curvilinear fits from GLME analysis along with $95 \%$ confidence intervals.

Abbreviations: CATS, correcting applanation tonometry surface; GAT, Goldmann applanation tonometer; GLME, general linear mixed-effects; IOP, intraocular pressure.

$p=0.04$ ). Figure 9 illustrates the independence of tear film adhesion to the measured applanation mire thickness using the PMMA-simulated corneas.

Fluorescein produces more tear film adhesion error than artificial tears by $0.51 \pm 0.38 \mathrm{mmHg}, p<0.001$, when measured using the PMMA hemisphere-simulated cornea. The difference using fluorescein and simulated tears was not significantly different in the cadaver eyes at $0.10 \pm 0.48 \mathrm{mmHg}, p>0.05$.

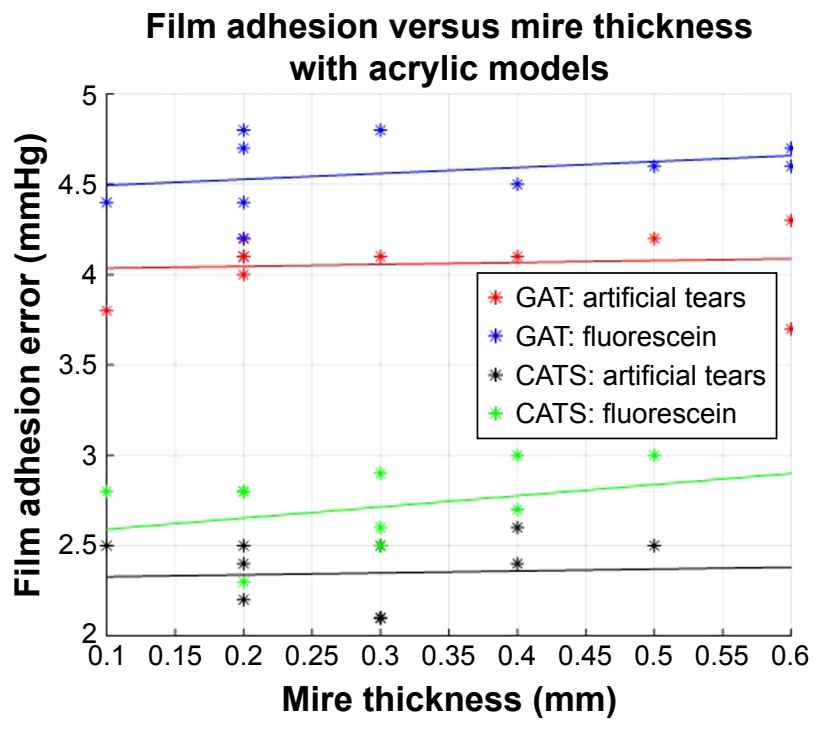

Figure 9 Tear film adhesion error versus applanation meniscus mire thickness in CATS and GAT prisms using GLME multivariate analysis output.

Abbreviations: CATS, correcting applanation tonometry surface; GAT, Goldmann applanation tonometer; GLME, general linear mixed-effects.

\section{Discussion}

The tear film adhesion force and resulting error in Goldmann applanation tonometry are clinically significant at an IOP underestimation of $4.57 \mathrm{mmHg}$. This IOP underestimation value resulting from the testing approximates the previously theorized IOP underestimation values of 4.1 and $3.3 \mathrm{mmHg} .{ }^{14,15}$ Tear film adhesion was originally thought to negate much of the corneal rigidity error seen with applanation tonometry giving a more or less accurate IOP measurement with GAT, which is true in part. ${ }^{6}$ However, the multiple error parameters related to corneal rigidity and the tear film error have considerable variability in individual patients, which leads to clinically significant errors in IOP measurement. ${ }^{2-7}$ The present study was designed to examine the isolated tear film adhesion error in Goldmann applanation tonometry. Other applanation tonometry error factors including $\mathrm{CCT}$, corneal curvature, and corneal rigidity (which encompassed CCT and the intrinsic corneal property of modulus of elasticity) were examined and published separately. ${ }^{6,7,14,20}$ The factors of CCT and corneal rigidity should not theoretically have an effect on the tear film adhesion error. Corneal curvature at the extremes could have an effect on tear film adhesion error by changing the contact angle theta $(\theta)$. The factors affecting tear film adhesion previously modeled and validated in this study include: ${ }^{14}$ 1) the contact angle theta $(\theta)$ between the tonometer and cornea over the tear film meniscus as illustrated in Figure 4 (upper right); 2) the circumference of the applanating tear film meniscus as illustrated in Figure 4 (lower right); and 3 ) the surface tension of the tear film.

The CATS tonometer prism significantly reduces GAT prism tear film adhesion error by $\sim 41 \%$. The testing results confirm previous mathematical modeling predicting a reduction of $45 \%$ in tear film error by increasing the contact angle between the applanating prism surface and the cornea over the tear film meniscus. ${ }^{14}$

Cadaver eye testing extrapolated to a zero IOP validates the approximate reduction in tear film adhesion force measured between the CATS and GAT prisms on the acrylic model. The separation of a prism from a manometrically fluidfilled cadaver eye simultaneously measures both the tear film adhesion and the IOP on the prism face over a dynamically reducing applanation area. Therefore, this dynamic process is not suitable to directly measure the isolated tear film separation at full applanation and indicates a falsely low separation force. For this reason, the static tear film separation conditions using the simulated corneas with PMMA hemispheres are likely more accurate. The $95 \%$ confidence intervals for the 
extrapolated mean tear film adhesion error to the $0 \mathrm{mmHg}$ IOP cadaveric eye do not overlap the $95 \%$ confidence intervals for mean film adhesion error from the acrylic cornea data. However, we do see that the second-order curvilinear fit does trend toward the film adhesion error values from the acrylic corneal model experiments as IOP approaches $0 \mathrm{mmHg}$. The results from this study encourage further investigation into the details of the process of film separation during tonometer applanation. It is possible that a second-order fit is not the most ideal fit for the data. Further mechanical analysis may justify other forms of statistical models.

There is some possible additional testing error using the PMMA hemispheres due to a relative hydrophobicity of the PMMA compared to the human cornea; however, this would likely only increase the error found in the human cornea. An applanating tonometer surface design conforming to the corneal curvature, producing a near-zero contact angle over the meniscus, may produce a significantly higher tear film adhesion error. The greatly increased adhesion with a zero contact angle would be similar to that seen with a hard contact lens on the cornea or comparable to two fluid-adhered microscope slides placed together requiring considerable separation force.

The thickness of the tear film meniscus seen in the imaged applanation mires appears to be independent of the tear film error. This independence is in contrast to common teaching about the Goldmann applanation technique, which most practitioners understand as thick mires can cause an overestimation in GAT measured IOP. The authors could locate no studies which support the correlation between the mire thickness and the IOP error or tear film adhesion. Previous mathematical modeling supports this independence. ${ }^{14}$ Fluids that have an increased surface tension such as fluorescein solution increase the tear film adhesion error. ${ }^{16-18}$ It is possible that the layered three-component tear film observed on the human eye may behave differently in its surface tension adhesive property than artificial tears. For the reasons stated earlier, we are unable to isolate the tear film adhesion from the IOP force on a live human eye, thus requiring the use of acrylic models and extrapolated cadaveric eye models.

\section{Acknowledgments}

The authors thank Georgia Eye Bank for assistance and Arizona Eye Consultants, Tucson, AZ, for extensive facilities use. The data sets used and/or analyzed during the current study are available from the corresponding author on reasonable request. The data will be available from our website www.Arizonaeyeconsultants.com at the time of publication. This study was supported in part by NIH SBIR Grant R43 EY026821-01 and Arizona Eye Consultants, Tucson, AZ.

\section{Disclosure}

Sean J McCafferty and Jim Schwiegerling have a vested interest in Intuor Technologies which owns the technology being tested in this manuscript. The authors report no other conflicts of interest in this work.

\section{References}

1. Susanna JR, De Moraes CG, Cioffi GA, Ritch R. Why do people (still) go blind from glaucoma? Transl Vis Sci Technol. 2015;4(2):1-10.

2. Liu J, Roberts C. Influence of cornea biomechanical properties on intraocular pressure measurement: quantitative analysis. J Cataract Refract Surg. 2005;31(1):146-155.

3. Kotecha A, Elsheikh A, Roberts C, Haogang Z, Garway-Heath D. Corneal thickness- and age-related biomechanical properties of the cornea measured with the ocular response analyzer. Invest Ophthalmol Vis Sci. 2006;47(12):5337-5347.

4. Whitacre M, Stein R. Sources of error with use of Goldmann-type tonometers. Surv Ophthalmol. 1993;38(1):1-30.

5. Neuburger M, Maier P, Böhringer D, Reinhard T, F Jordan J. The impact of corneal edema on intraocular pressure measurements using Goldmann applanation tonometry, Tono-Pen XL, iCare, and ORA: an in vitro model. J Glaucoma. 2013;22(7):584-590.

6. McCafferty S, Lim G, Duncan W, et al. Goldmann tonometer error correcting prism: clinical evaluation. Clin Ophthalmol. 2017;11:835-840.

7. McCafferty S, Levine J, Schwiegerling J, Enikov E. Goldmann applanation tonometry error relative to true intracameral Intraocular pressure in vitro and in vivo. BMC Ophthalmol. 2017;17(1):215.

8. Kass M, Heuer D, Higginbotham E, et al. The Ocular Hypertension Treatment Study: a randomized trial determines that topical ocular hypotensive medication delays or prevents the onset of primary openangle glaucoma. Arch Ophthalmol. 2002;120(6):701-713.

9. Brandt J, Gordon M, Gao F, Beiser J, Phillip J. Adjusting intraocular pressure for central corneal thickness does not improve prediction models for primary open-angle glaucoma. Ophthalmology. 2012;119(3): 437-442.

10. Feltgen N, Leifert D, Funk J. Correlation between central corneal thickness, applanation tonometry, and direct intracameral IOP readings. Br J Ophthalmol. 2001;85(1):85-87.

11. Kniestedt C, Nee M, Stamper R. Dynamic contour tonometry: a comparative study on human cadaver eyes. Arch Ophthalmol. 2004; 122(9):1287-1293.

12. Eisenberg D, Sherman B, Mckeown C, Schuman J. Tonometry in adults and children: a manometric evaluation of pneumotonometry, applanation, and tonopen in vitro and in vivo. Ophthalmology. 1998;105(7): $1173-1181$.

13. Riva I, Quarantra L, Russo A, Katsanos A, Rulli E, Floriani I. Dynamic contour tonometry and Goldmann applanation tonometry: correlation with intracameral assessment of intraocular pressure. Eur J Ophthalmol. 2012;22:55-62.

14. McCafferty S, Lim G, Duncan W, Enikov E, Schwiegerling J. Goldmann tonometer prism with an optimized error correcting applanation surface. Transl Vis Sci Technol. 2016;5(5):1-5.

15. Damji K, Muni R, Munger R. Influence of corneal variables on accuracy of intraocular pressure measurement. J Glaucoma. 2003;12:69-80.

16. Tselishchev Y, Val'tsifer V. Influence of the type of contact between particles joined by a liquid bridge on the capillary cohesive forces. Colloid J. 2003;65:385-389.

17. Orr F, Scriven L, Rivas A. Pendular rings between solids: meniscus properties and capillary force. J Fluid Mech. 1975;67:723-742. 
18. Zeng Y, XinXing G, Jun L, Xing L. Effect of tear film changes on the intraocular pressure measurement by Goldmann applanation tonometer. Eye Sci. 2008;24(1):27-29.

19. Tang J, Pan X, Weber P, Liu J. Effect of corneal stiffening on Goldmann Applanation Tonometery and Tonopen measurement in canine eyes. Invest Ophthalmol Vis Sci. 2012;53(3):1397-1405.
20. McCafferty S, Levine J, Schwiegerling J, et al. Goldmann and error correcting tonometry prisms compared to intracameral pressure. Clin Ophthalmol. 2017;11:835-840.

\section{Publish your work in this journal}

Clinical Ophthalmology is an international, peer-reviewed journal covering all subspecialties within ophthalmology. Key topics include: Optometry; Visual science; Pharmacology and drug therapy in eye diseases; Basic Sciences; Primary and Secondary eye care; Patient Safety and Quality of Care Improvements. This journal is indexed on

\section{Dovepress}

PubMed Central and CAS, and is the official journal of The Society of Clinical Ophthalmology (SCO). The manuscript management system is completely online and includes a very quick and fair peer-review system, which is all easy to use. Visit http://www.dovepress.com/ testimonials.php to read real quotes from published authors. 